\title{
Quantified patient preferences for lifestyle intervention programs for diabetes prevention-a protocol for a systematic review
}

\author{
Charalabos-Markos Dintsios $^{1^{*}}$ (D), Nadja Chernyak', Benjamin Grehl ${ }^{1}$ and Andrea Icks ${ }^{1,2,3}$
}

\begin{abstract}
Background: The 20-70\% participation of diabetes patients in lifestyle interventions (LSI) worldwide seems to be rather sub-optimal, in spite of all intents of such interventions to delay further progress of the disease. Positive effects through LSI are expected in particular for patients who suffer less from diabetes-related limitations or other chronic diseases. Seeing that diabetes prevalence and with it mortality are increasing, LSI have become an inherent part of diabetes treatment standards. Various qualitative studies have been carried out to identify participation barriers for LSI. However, these have not resulted in more detailed knowledge about the relative importance of factors with an inhibiting impact on participation. Since it cannot be assumed that all of the influencing factors have equivalent values, it is necessary to investigate their individual importance with regard to a positive or negative decision about participating. There are no systematic reviews on patient preferences for LSI programs in diabetes prevention. As a result, the main objectives of this systematic review are to (i) identify existing patient preference elicitation studies related to LSI for diabetic patients, (ii) summarize the methods applied and findings, and (iii) appraise the reporting and methodological quality of such studies.

Methods: We will perform systematic literature searches to identify suitable studies from 14 electronic databases. Retrieved study records will be included based on predefined eligibility criteria as defined in this protocol. We will run abstract and full-text screenings and then extract data from all selected studies by filling in a predefined data extraction spreadsheet. We will undertake a descriptive, narrative synthesis of findings to address the study objectives, since no pooling for quantified preferences is for methodological reasons implementable. We will pay special attention to aspects of methodological quality of preference elicitation by applying established evaluation criteria of the ISPOR and some own developed criteria for different elicitation techniques. All critical stages within the screening, data extraction, and synthesis processes will be conducted by two pairs of authors. This protocol adheres to PRISMA and PRISMA-P standards.
\end{abstract}

Discussion: The proposed systematic review will provide an overview of the methods used and current practice in the elicitation and quantification of patients' preferences for diabetes prevention lifestyle interventions. Furthermore, the methodological quality of the identified studies will be appraised as well.

\section{Systematic review registration: PROSPERO CRD42018086988}

Keywords: Diabetes mellitus, Patient preferences, Preferences weights, Preference elicitation, Conjoint analysis, Discrete choice analysis, Analytic hierarchy process, Multi-criteria decision analysis, Systematic review

\footnotetext{
* Correspondence: dintsios@hhu.de

${ }^{1}$ Institute for Health Services Research and Health Economics, Centre for

Health and Society, Faculty of Medicine, Heinrich-Heine University

Düsseldorf, Moorenstr. 5, 40255 Düsseldorf, Germany

Full list of author information is available at the end of the article
}

(c) The Author(s). 2018 Open Access This article is distributed under the terms of the Creative Commons Attribution 4.0 International License (http://creativecommons.org/licenses/by/4.0/), which permits unrestricted use, distribution, and

reproduction in any medium, provided you give appropriate credit to the original author(s) and the source, provide a link to the Creative Commons license, and indicate if changes were made. The Creative Commons Public Domain Dedication waiver (http://creativecommons.org/publicdomain/zero/1.0/) applies to the data made available in this article, unless otherwise stated. 


\section{Background}

\section{Disease burden}

Diabetes mellitus is a highly prevalent chronic disease affecting approximately 9 to $10 \%$ of the global adult population [1]. Based on estimates of the International Diabetes Federation, there were 425 million adults with diabetes in 2017, and this number is expected to rise to 629 million by 2045 [2]. Despite several efforts to improve diabetes care, the disease is still associated with markedly increased morbidity and mortality as well as high cost for the healthcare system [2-5]. The treatment of diabetes mellitus as a chronic disease depends on patient willingness to adopt changes in lifestyle, nutrition, and therapy self-management [6].

\section{Lifestyle interventions}

Lifestyle interventions (LSI) are a central part of diabetes care, in particular in type 2 diabetes mellitus [7-9]. It has been shown that LSI can improve HbA1c and blood pressure [10], slow down the progression of the disease and reduce late complications [11-14], or increase quality of life even if not showing significant long-term differences in cardiovascular morbidity and mortality [15]. The beneficious effect of LSI has been verified, particularly for physical activity, in prediabetes as well, as shown in a recent meta-analysis, improving oral glucose tolerance (risk ratio [RR] $-0.26,95 \% \mathrm{CI}-0.06$ to 0.07 ) and fasting blood glucose (RR $-0.05,95 \%$ CI -0.14 to 0.04 ) [16]. Furthermore, LSI can added to usual medical care even lead to modest cost savings [17]. Program intensity seems to play a major role in weight loss outcomes, but programs that have high uptake can still have considerable impact in lowering diabetes risk in a population, even with a low-intensity intervention [18]. Furthermore, it seems that LSI are readily scalable and exportable in primary care with potential for substantial clinical and public health impact [19]. Positive effects through LSI are expected in particular for patients between the ages of 35 and 65 years who, in comparison to older patients, suffer less from diabetes-related limitations or other chronic diseases [20]. For this reason, LSI are directed particularly at this age group. In an umbrella review on good practice characteristics of diet and physical activity interventions and policies, the use of theory, participants, target behavior, content development/management, multidimensionality, practitioners, and settings were identified as being relevant for the intervention; participation processes, training for practitioners, the use/integration of existing resources, feasibility, maintenance/sustainability, implementation partnerships, implementation consistency/adaptation processes, and transferability were the important implementation characteristics [21]. However, a 20-70\% participation in LSI in general is low [22-24]. Evidence on the impact of incentives to promote LSI remains sparse or as exemplarily shown for physical activity prescriptions of low methodological quality [25].

The choice of particular clinical goals and selection of lifestyle and pharmacologic treatments to reach those goals is a multifaceted process that requires using valid and reliable methods that transparently report on data elicitation processes, potential strengths and shortcomings, and efficiently eliciting patient preferences and values as well by integrating these with evidence of treatment effectiveness as well as provider preferences [26]. Various qualitative studies have been carried out to identify participation barriers for LSI, such as costs and small portion sizes in diet, missing support of family and private issues, strict diet being similarly burdensome to insulin intake, reduced quality of life for dietary interventions, risk of hypoglycemia, work schedule, fear of being tired, low fitness level for physical activity interventions, and finally, psychological distress in general [27-31]. However, these have not resulted in more detailed knowledge about the relative importance of factors with an inhibiting impact on participation. Since it cannot be assumed that all of the influencing factors have equivalent values, it is necessary to investigate their individual importance with regard to a positive or negative decision about participating. In the past, it has been possible to determine the individual importance of various factors relating to participating in such interventions but with a different target population [32, 33]. This raises the question about the quantification of patient preferences for LSI programs in diabetes prevention, especially considering that individual preferences for changes over a LSI are linked with respective outcomes [33]. Tailoring programs rather than using a generic "one size-fits-all" approach should be implemented given some of the observed differences within demographic groups, social-cognitive and behavior constructs with physical activity counseling, and programming preferences. Meeting the needs, interests, motivation, and level of behavior may help to increase physical activity adherence over time. This means developing specific programs for a variety of settings, intensities, structures, and types of activity [31].

\section{Preferences}

Preferences are comparative judgements between entities [34]. In this sense, a preference is the choice of one thing over another with the anticipation that the choice will result in greater value, satisfaction, capability, or improved performance of the individual, the organization, or the society. From a patient perspective, preference instruments reveal what is relevant to the 
patient and can support decisions, which directly or indirectly affect the patient. They may include even non-health outcomes of health interventions [35]. Interestingly, patient preferences can differ compared to physicians' judgment, as shown in different systematic reviews [36, 37]. Most studies revealed a disparity between the preferences of actual patients and those of physicians. For most conditions, physicians underestimated the impact of intervention characteristics on patients' decision-making. Especially for type 2 diabetes mellitus (T2DM) medication treatment, there seems to be a high preference agreement for the main health benefits, but the order between patients and physicians differed [38]. But even transferred to the research agenda, views of diabetic patients and their relatives regarding their preferred research fields may differ when compared to current scientific activity in diabetology, leading to a plea to involve diabetic patients and their relatives in the weighting and selection of research topics more often $[39,40]$.

Health preference research is connected with the following assumptions [41]: (i) it can be used to elicit patient preferences on intervention attributes, and the resulting preference estimates are valid and reliable; (ii) it can be used to improve the design and implementation of health; (iii) designing health interventions to better meet the needs of patients not only improves patient satisfaction, but also improves patient behaviors such as uptake and adherence; and finally (iv) improvements in patient satisfaction, uptake, and adherence as a result of health preference research lead to better health and, ultimately, to more efficient healthcare systems interventions. Since patient welfare is presumably an objective of providing healthcare services, patient values should according to Mühlbacher and Johnson [42] logically play a central role in approval, utilization, reimbursement, and pricing decisions. To give patients' preferences appropriate roles in the treatment life cycle, a publicprivate research initiative has recently been launched [43]. Considering patients' preferences in individual decisions concerning prevention, diagnostics, or therapy has become more and more important (e.g., shared decision-making). Especially, diabetes treatment requires patients to have significant participation in their care and self-management to achieve glycemic control [44]. It has been shown that this may lead to higher satisfaction with the communication and higher acceptance of and adherence to therapy and, thereby, even to improved health outcomes $[41,45,46]$.

\section{Preference elicitation}

In a systematic review to identify the use of preference elicitation methods in healthcare decision-making [47], studies applied different elicitation methods with various degrees of complexity. Among others, multi-criteria decision analysis (MCDA) has been used to quantify and capture patients' preferences [48].

The International Society for Pharmacoeconomics and Outcomes Research (ISPOR) adopted a broad approach for MCDA, including in the consideration of MCDA methods that help deliberative discussions using explicitly defined criteria. A pragmatic definition is given by Mühlbacher and Kaczynski [49]: "MCDA can be seen as a tool to incorporate objective and subjective measures in a transparent decision-making process that identifies and weighs multiple evaluation criteria in order to prioritize different treatment strategies or health technologies." The value of MCDA lies not only in the results it generates, but also in its capacity to display the logical connection between inputs (i.e., data and assumptions) and outputs in the form of valued consequences (and costs). A MCDA should seek to model as simple as possible, yet sufficiently complex to incorporate all relevant aspects and their values. In general, value measurement, outranking, and goal programming are used in practice as MCDA approach implementing different techniques for decision support. Two reviews show that MCDA has been applied to a broad range of areas in the health care with the use of a variety of methodological approaches [50, 51]. The authors conclude that further research on MCDA techniques should include the development of guidelines on the assumptions underlying different approaches and their practical implications for decision-makers, and further testing of the impact of different MCDA approaches on decision-making. Simplicity and transparency of a MCDA are an aid to understanding the conclusion for decision-makers and other potential users of the analysis. This is challenging when exploring the complexity of MCDA techniques such as conjoint analysis (CA) or discrete-choice experiments (DCE), analytic hierarchy process (AHP), best-worst scaling (BWS), point allocation, swing weights approach, and other defined scales. Of these techniques, DCE is a widely accepted approach to eliciting stated preferences for health outcomes and processes. The important advantage of choice experiments is their foundation in microeconomic utility theory [52]. The use of DCE in healthcare continues to grow dramatically, as does the scope of applications across an expanding range of countries [53] accompanied by a shift towards statistically more efficient designs and flexible econometric models [54]. Furthermore, DCE can produce reasonable predictions of health-related behaviors [55] and has shown a high predictive value for actual behavior in a public health setting [56]. AHP seems also to play a more important role in medical and healthcare decision-making [57] and has been especially used to elicit patient preferences $[58,59]$ as well as for medication decision-making in T2DM with experts (Additional file 1)[60]. 


\section{Preference studies}

A preceded feasibility search determined a plethora of studies and some scoping or systematic reviews concerning patient preferences in connection with various medication interventions for diabetes disease [61-67]. It should be noted that there are plenty of available pharmaceutical therapies for diabetes [68]. Yet, in the feasibility search, there were identified a few studies that had been conducted specifically on patient preferences for LSI $[69,70]$. Apart from the intervention-relevant influence factors with regard to participating in or designing LSI, these survey studies on patient preferences have also partly examined the willingness to pay and the design of the financial incentives for participating in the programs offered. Each study made reference to the corresponding national healthcare context.

\section{Study objective and rationale}

While there are systematic reviews regarding treatment preferences in people with diabetes, a systematic review adressing LSI preferences is mising. The main objective of this systematic literature review is to identify existing studies eliciting patient preferences for LSI programs for diabetes prevention, summarize the methods applied and findings, and appraise their methodological quality. We will review identified studies with regard to their methodical quality to provide an overview of current practices in patient preference elicitation for LSI programs in diabetes prevention reported in the literature and to find out which program aspects are included and how important they are for surveyed patients and professionals. Since pooling preferences elicited with different methods in various samples is not applicable, we will summarize the findings of the identified studies by narrative comparisons.

\section{Methods \\ Protocol}

This protocol adheres to the Preferred Reporting Items in Systematic Reviews and Meta-analyses (PRISMA) statement [71] and PRISMA for systematic review protocols (PRISMA-P) statement [72]. The PRISMA-P checklist is given in Additional file 2. The protocol is registered in the International Prospective Register of Systematic Reviews (PROSPERO) CRD42018086988.

\section{Eligibility criteria}

We will include studies reporting quantified elicitation of preferences on lifestyle programs for diabetes prevention with characteristics given in Table 1 . We will exclude studies considering only qualitative and not quantitative preference elicitation. Qualitative approaches follow their own methodology. Their identification and the appraisal of the implemented methods within qualitative studies constitute an own topic in itself which should be captured in a specific systematic review.

\section{Information sources}

The following literature databases will be searched: PubMed (via NLM), MEDLINE (via Ovid), Journals@Ovid

Table 1 Eligibility criteria in study selection

\begin{tabular}{|c|c|c|}
\hline Criteria & Inclusion & Exclusion \\
\hline Population & - Patients & $\begin{array}{l}\text { - Patients suffering from other conditions } \\
\text { than diabetes } \\
\text { - Professionals (physicians, nurses, etc.) }\end{array}$ \\
\hline Condition & $\begin{array}{l}\text { - T1DM and T2DM } \\
\text { - All stages of diabetes disease } \\
\text { - Complications of diabetes } \\
\text { - Metabolic disorders in relation with diabetes }\end{array}$ & - Conditions unrelated to diabetes \\
\hline Intervention & $\begin{array}{l}\text { - Quantitative elicitation of preferences } \\
\text { for lifestyle interventions } \\
\text { - All available quantitative methods for stated } \\
\text { preferences (MCDA, MADM, MODM, CA, DCE, } \\
\text { AHP, BWS, WTP, etc.) }\end{array}$ & $\begin{array}{l}\text { - Qualitative elicitation of preferences for } \\
\text { lifestyle interventions } \\
\text { - Quantitative or qualitative elicitation of } \\
\text { preferences exclusively for pharmacological } \\
\text { treatment of diabetes } \\
\text { - Elicitation of utilities }\end{array}$ \\
\hline Study design & $\begin{array}{l}\text { - The study is based on single and/or multiple } \\
\text { data sets }\end{array}$ & \\
\hline Type of publication & $\begin{array}{l}\text { - Publications in peer-reviewed journals which } \\
\text { can be retrieved through our search approach } \\
\text { - Studies, to which full publication is available }\end{array}$ & $\begin{array}{l}\text { - Editorials, comments, newspaper articles, and } \\
\text { other forms of popular media } \\
\text { - Conference abstracts } \\
\text { - Studies, to which no full publication is available }\end{array}$ \\
\hline Date & - No restriction & \\
\hline Language & $\begin{array}{l}\text { - No restrictions on language, but available } \\
\text { English, French, Spanish, or German abstract is } \\
\text { a pre-condition for inclusion }\end{array}$ & $\begin{array}{l}\text { - No English, French, Spanish, or German abstract } \\
\text { available }\end{array}$ \\
\hline
\end{tabular}


(via Ovid), EMBASE (via Ovid), EconLit (via EBSCOhost), Web of Science (via Thomson Reuters), Science direct (via Elsevier), and PsycINFO (via Ovid).

To identify potential existing systematic reviews with regard to our research topic, we will also search the Cochrane Library (via Wiley) including the NHS CRD databanks Database of Abstracts of Reviews of Effects (DARE), Health Technology Assessment (HTA), and NHS Economic Evaluation Database (NHS EED). We will also include grey literature databases in our search: CINAHL (via EBSCOhost), Catalogue ZB MED (via ZBMed), Current Contents (via ZBMed), DissOnline (via ZBMed), Econis (via ZBMed), and Econbiz (via $\mathrm{ZBW}$ ) and finally for the German region the "Karlsruher virtueller Katalog" as well.

\section{Search strategy}

We will not restrict the timeframe of the search nor will we apply any search filters [73] to guarantee for high sensitivity irrespectively of the respective specificity as a result of our feasibility search which yielded only two relevant hits. We composed the "primary" bibliographic search strategy according to PubMed search rules, which will be translated to other database syntaxes. We have applied the search strategy to the relevant studies found in the previous feasibility and known in the research field to check for the search strategy's sensitivity. The details of this search strategy are given in Table 2.

\section{Technical tools}

The hits of the systematic literature search will be collected and stored in EndNote (Version $\times$ Patient preferences versus physicians7, Thompson Reuters). The abstract and full-text screening will be carried out using the EndNote Surface. During the data extraction, we will fill in our own spreadsheet forms. All information will be shared and be made available within the working group consisting of two tandems.

\section{Study selection}

We will select studies in several steps. First, we will identify all relevant studies by conducting the search in the scientific publication databases listed in the "Information sources" section. We will remove duplicates to reduce the reviewers' workload associated with the next steps. Next, we will screen titles and abstracts of all references selected so far. Two tandems of researchers will independently review and include or exclude references guided by the eligibility criteria mentioned above. The title and abstract screening will be piloted first within the tandems independently and then between the tandems to adjust the procedure. Any disagreement and inconsistency in the final decisions will be resolved in the further discussion among all researchers involved in the screening. Full-text screening will be the final step within our study selection process. Four independent researchers (the members of the two tandems) will review the full texts of the studies selected in the previous step to collect the final list of studies for data extraction. The decision on inclusion or exclusion will be made on the basis of the eligibility criteria. Any apparent discrepancies appearing during the full-text screening will be resolved by discussion among the researches. The screening process will be reported accurately and in sufficient detail-including the title and abstract screening and the full-text screening-to complete a PRISMA flowchart. The number of the excluded studies accompanied by respective exclusion reasons will be recorded. Flowcharts for included and excluded studies will be provided.

\section{Additional data source selection}

During the title/abstract screening, we will collect systematic literature reviews addressing LSI in diabetes prevention as a potential reference source of interest. After the full-text screening, we will export the reference list of all publications identified for the data extraction. Moreover, we will check forward citations of these publications by tracking them in different citation databases. We will apply PubMed's "related articles" feature for the same papers to collect the first 10 hits. The restricted number of hits is voluntarily chosen to reduce the workload. All references gathered in this step will be subject to the same steps as described in the "Study selection" section.

\section{Data extraction}

Two reviewers will independently extract data from all included studies after the full-text review by filling in a predefined data extraction spreadsheet. The data extraction spreadsheet was first piloted. The data extraction spreadsheet is provided in addition to the paper documents (Additional file 1). Data to be extracted will be arranged into the following categories:

1. Research question and sample

2. Perspective of preference elicitation

3. Method of preference elicitation

4. Method of preference quantification and authors' rationale for its choice

5. Criteria, attributes, and values of preferences

6. Subgroup analysis (as pre-specified in the studies)

7. Results of preference quantification

\section{Quality appraisal}

The data extraction sheet contains questions and categories that are related to the methodological quality appraisal of the patient preference elicitation. These 
Table 2: Search strategy for MEDLINE (via PubMed)

\begin{tabular}{|c|c|c|}
\hline \multicolumn{2}{|c|}{ Databank } & PubMed (NLM) \\
\hline \multicolumn{2}{|c|}{ Timespan: } & Unlimited \\
\hline \multicolumn{2}{|c|}{ Language: } & Unrestricted \\
\hline \multicolumn{2}{|c|}{$\begin{array}{l}\text { Date of } \\
\text { creation: }\end{array}$} & 09 September 2017 \\
\hline \# & Hits & Query \\
\hline$\# 1$ & 64,223 & "DIABETES MELLITUS, TYPE 1"[MESH] \\
\hline \#2 & 95,093 & "DIABETES MELLITUS, TYPE 2"[MESH] \\
\hline$\# 3$ & 7315 & "DIABETES INSIPIDUS"[MESH] \\
\hline$\# 4$ & 34,268 & $\begin{array}{l}\text { "INSULIN/ADMINISTRATION AND DOSAGE"[MESH] } \\
\text { OR "INSULIN/ECONOMICS"[MESH] OR "INSULIN/ } \\
\text { ORGANIZATION AND ADMINISTRATION"[MESH] OR } \\
\text { "INSULIN/THERAPEUTIC USE"[MESH] OR "INSULIN/ } \\
\text { THERAPY"[MESH] }\end{array}$ \\
\hline$\# 5$ & 256,585 & $\begin{array}{l}\text { (diabetes [ti] OR diabetic [ti] OR T1DM [ti] OR T2DM } \\
\text { [ti] OR NIDDM [ti]) }\end{array}$ \\
\hline$\# 6$ & 311,637 & $\# 1$ OR \#2 OR \#3 OR \#4 OR \#5 \\
\hline \#7 & 8688 & "RISK REDUCTION BEHAVIOR"[MESH] \\
\hline$\# 8$ & 69,754 & "LIFE STYLE"[MESH] \\
\hline$\# 9$ & 134,304 & "EXERCISE"[MESH] \\
\hline$\# 10$ & 96,110 & "SPORTS"[MAJR] \\
\hline$\# 11$ & 17,011 & "REHABILITATION"[MESH:NOEXP] \\
\hline$\# 12$ & 4582 & “COMMUNITY HEALTH PLANNING"[MESH] \\
\hline \#13 & 142,616 & "HEALTH EDUCATION"[MESH] \\
\hline$\# 14$ & 59,790 & “HEALTH PROMOTION"[MESH] \\
\hline \#15 & 499 & $\begin{array}{l}\text { change*[ti] AND diabetes[tiab] AND (diet[ti] OR } \\
\text { behavior*[ti] OR behaviour*[ti] OR nutrition [ti] OR } \\
\text { activity [ti] OR activities [ti] OR training [ti] OR physical } \\
\text { [ti] OR athletic [ti]) }\end{array}$ \\
\hline \#16 & $1,191,618$ & $\begin{array}{l}\text { (lifestyle [tiab] OR life style [tiab] OR behaviour*[ti] OR } \\
\text { diet program*[tiab] OR behavior*[ti] OR exercise [ti] OR } \\
\text { training program*[tiab] OR preventive strategy [tiab] OR } \\
\text { prevention program*[tiab] OR behavioral prevention } \\
\text { [tiab] OR behavioral program*[tiab] OR way of life [tiab] } \\
\text { OR manner of living [tiab] OR behavioral intervention* } \\
\text { [tiab] OR diet [ti] OR behavioral intervention*[tiab] OR } \\
\text { community program*[tiab] OR nutrition [ti] OR activity } \\
\text { [ti] OR activities [ti] OR training [ti] OR physical [ti] OR } \\
\text { athletic [ti]) }\end{array}$ \\
\hline$\# 17$ & $2,779,775$ & $\begin{array}{l}\# 7 \text { OR \#8 OR \#9 OR \#10 OR \#11 OR \#12 OR } 13 \\
\text { OR \#14 OR \#16 }\end{array}$ \\
\hline$\# 18$ & 47,980 & \#6 AND \#17 \\
\hline$\# 19$ & 48,163 & $\# 15$ OR \#18 \\
\hline$\# 20$ & 17 & $\begin{array}{l}\text { \#19 AND (conjoint [tiab] OR discrete choice } \\
\text { [tiab] OR multi-criteria*[tiab] OR multicriteria*[tiab] } \\
\text { OR multi-attribute*[tiab] OR multiattribute }{ }^{*}[\text { tiab] OR } \\
\text { multi-objective*[tiab] OR multiobjective*[tiab] OR } \\
\text { multi-alternative }{ }^{*}\left[\text { tiab] OR multialternative }{ }^{*}[\text { tiab] OR }\right. \\
\text { multiple criteria[tiab] OR multiple attribute }{ }^{*}[\text { tiab] OR } \\
\text { multiple objective*[tiab] OR multiple alternative*[tiab]) }\end{array}$ \\
\hline
\end{tabular}

\#21 $24 \quad$ \#19 AND (stated preference*[tiab] OR willingness to pay [tiab] OR willing to pay [tiab] OR wtp [tiab] OR analytic hierarchy process*[tiab] OR ahp [tiab])

\#22 218 \#19 AND (decision-analy*[tiab] OR decision-making [tiab] OR patient preference*[tiab] OR patient* prioriti*[tiab]
Table 2: Search strategy for MEDLINE (via PubMed) (Continued) OR (patient [ti] AND prioritizing[ti]) OR elicit* patient* [tiab] OR preference* ${ }^{*}[\mathrm{ti}]$ OR health-state utilit*[tiab] OR $\left.E Q-5 D^{*}[t i]\right)$

\#23 15 \#19 AND (health state utilit* OR utility measure*[tiab] OR health state utilit*[tiab] OR utility value*[tiab] OR utility score*[tiab] OR utility-based score*[tiab] OR health state valuation [tiab] OR m-mauf*[tiab])

\#24 19 \#19 AND (hui2 [tiab] OR hui3 [tiab] OR hui-2 [tiab] OR hui-3 [tiab] OR health utility [tiab] OR health utilities [tiab])

\#25 $0 \quad$ \#19 AND (part-worth [tiab] OR paired comparisons [tiab] OR pairwise choices [tiab] OR pairwise choice [tiab])

\#26 $97 \quad$ \#19 AND (decision-analy*[ti] OR decision-making [ti] OR decision support*[ti] OR preference*[ti] OR prioriti*[ti] OR ((choice*[ti] OR elicit*[ti]) AND patient*[i] ))

$2715 \quad \# 19$ AND (best-worse scaling [ti] OR preference weight [ti] OR willingness to accept [ti] OR out-of-pocket[ti] OR mcda [ti] OR trade-off[ti] OR utilities [ti] OR valuation [ti])

\#19 AND (goal programming OR vector optimization [tiab] OR value theory [tiab] OR maut [tiab] OR mavt [tiab] OR promethee [tiab] OR preference ranking organization [tiab] OR method for enrichment evaluations [tiab] OR electre [tiab] OR elimination et choix traduisant la realite [tiab])

\#29 0 \#19 AND (mental modeling [tiab] OR trade-off analysis [tiab] OR tradeoff analysis [tiab] OR risk-based prioritization [tiab])

\#30 0

\#19 AND (((gaia[tiab] OR meteor [tiab] OR kepner-tregoe [tiab] OR archimedean [tiab] OR tchebycheff [tiab] OR min-max [tiab] OR step method [tiab] OR interactive multiple goal programming [tiab] OR imgp [tiab] OR priam [tiab] OR scalarizing function [tiab] OR outranking concept [tiab] OR goal point approach*[tiab] OR reference point approach* ${ }^{*}[$ tiab] OR concordance index [tiab] OR concordance indices[tiab] OR discordance indices [tiab] OR discordance index [tiab] OR trade-off term*[tiab] OR trade-off terms [tiab] OR voting weight* [tiab] OR fuzzy set theory [tiab] OR fuzzy sets [tiab] OR correspondence analysis [tiab]) AND (decision-making [tiab] OR decision-analy*[tiab]))

\#31 $1 \quad 25,151,503$ [pmid]

\#32 $103 \quad$ Similar articles for PubMed (Select 25,151,503)

\#33 $125,348,049$ [pmid]

\#34 103 Similar articles for PubMed (Select 25,348,049)

\#35 $57 \quad$ \#34 AND (diabetes OR diabetic OR insulin*)

\#36 $1 \quad 24,289,831$ [pmid]

\#37 188 Similar articles for PubMed (Select 24,289,831)

\#38 $55 \quad$ \#37 AND (diabetes OR diabetic OR insulin*)

\#39 $199 \quad$ \#32 OR \#35 OR \#38

\#40 $509 \quad$ \#20 OR \#21 OR \#22 OR \#23 OR \#24 OR \#25 OR \#26 OR \#27 OR \#28 OR \#29 OR \#30 OR \#32 OR \#35 OR \#38

parameters are based among others on different ISPOR task forces (ISPOR MCDA Emerging Good Practices [48, 74], ISPOR Conjoint Analysis Good Research Practices [75], ISPOR Good Research Practices for Conjoint Analysis [76], and ISPOR Conjoint Analysis Experimental 
Design Good Research Practices [77]) and other materials or checklists discussing the quality of preference elicitation methods [54, 61, 78, 79] completed in addition by own quality criteria, especially for AHP. The quality appraisal includes furthermore the assessment of the appropriateness of the chosen method for the specific research question and the subsequently derived criteria or attributes for the implemented preference elicitation. Additionally, it will be checked if preference elicitations are accompanied by qualitative research since validity and reliability of preference estimators are despite methodological elicitation appropriateness still at stake. If information is not provided, we will assign "not applicable," "not reported," or "not enough information" to the corresponding questions.

\section{Data synthesis}

Since patient preference elicitation methods are different and the preference measures are largely diverse, we will concise results in a narrative descriptive analysis summarizing them according to the implemented approaches and contrasting the rankings of comparable attributes or criteria. The latter will be done by clustering respective attributes or criteria according to their type and subsequently by comparing the order and the weights of the ranked attribute or criteria clusters.

\section{Discussion}

This protocol describes objectives, methods, and steps of an upcoming systematic review that will include studies on the quantification of patient preferences for LSI in diabetes prevention. To the best of our knowledge, this contribution to the scientific community is novel since no publications are specially targeting the elicitation of patient preferences for lifestyle interventions in diabetes prevention systematically. The objectives are to identify studies eliciting patient preferences for lifestyle intervention programs for diabetes prevention, appraise their methodological quality and their reporting, and finally summarize findings to provide an overview of the current practice in this field.

\section{Additional files}

Additional file 1: Data extraction spreadsheet. (DOCX $23 \mathrm{~kb}$ )

Additional file 2: PRISMA-P checklist. (DOCX $37 \mathrm{~kb}$ )

\section{Abbreviations}

AHP: Analytic hierarchy process; BWS: Best-worst scaling; CA: Conjoint analysis; CDSR: The Cochrane Database of Systematic Reviews; CENTRAL: Cochrane Central Register of Controlled Trials; CINAHL: Cumulative Index to Nursing and Allied Health Literature; DARE: The Database of Abstracts of Reviews of Effects; DCE: Discrete-choice experiment: DissOnline: Dissertation online; DM: Diabetes mellitus; Econbiz: Academic search portal provided by the German National Library of Economics Leibniz Information Centre for Economics; Econis: Academic search portal provided by the German National Library of Economics - Leibniz Information Centre for Economics; EconLit: Economic literature search portal provided by the American Economic Association; EMBASE: Excerpta Medica database; HbA1c: Glycated hemoglobin; HTA: Health technology assessment; IDF: International Diabetes Federation; ISPOR: The International Society for Pharmacoeconomics and Outcomes Research; LSI: Lifestyle interventions; MCDA: Multi-criteria decision analysis; MEDLINE: Medical Literature Analysis and Retrieval System Online; NHS CRD: NHS Centre for Reviews and Dissemination; NHS EED: NHS Economic Evaluation Database; NHS: National Health Service; NLM: US National Library of Medicine; PRISMA: The Preferred Reporting Items in Systematic Reviews and Meta-analyses; PRISMA-P: The Preferred Reporting Items in Systematic Reviews and Meta-analyses for Protocols; PROSPERO: The International Prospective Register of Systematic Reviews; PsycINFO: Psychological literature search portal provided by the American Psychological Association; T2DM: Type 2 diabetes mellitus; ZB MED: German Central Library for Medicine

\section{Acknowledgements}

We are thankful to Sigrid Droste who contributed substantially to the formulation of the search strategies.

\section{Funding}

This study received no funding sources by any public or private institution.

\section{Availability of data and materials}

Not applicable

\section{Protocol amendments}

If the present protocol is substantially amended after an initiation that may impact on the conduct of the study (including eligibility criteria, study objectives, study design, study analysis), then this amendment will be agreed upon by all collaborators prior to the implementation and will be documented in a note to a later publication or a report (section "Differences between protocol and review").

\section{Authors' contributions}

All authors made substantial contributions to the conception and design of the study and reviewed all documents and materials. CMD was responsible for the development of the study design as well as the formulation of the study question, the selection criteria, and the search terms. NC, BG, and Al peer-reviewed and amended the search strategy and the data extraction sheets. The screening and selection of relevant hits was implemented by two groups (CMD and $\mathrm{BG}$ ) and (NC and $\mathrm{Al})$. All authors were responsible for compiling the table and the data extraction spreadsheet. CMD drafted the first manuscript, and all the authors contributed to the manuscript revision before the final version. All authors read and approved the final manuscript. CMD is the guarantor of the review.

\section{Authors' information}

$\mathrm{CMD}$ is, next to his industrial affiliation, a visiting researcher at the Institute for Health Services Research and Health Economics at the Heinrich-Heine-University, Düsseldorf, Germany. NC is a senior researcher at the Institute for Health Services Research and Health Economics at the Heinrich-Heine-University, Düsseldorf, Germany. BG is a cooperating psychologist at the Institute for Health Services Research and Health Economics at the Heinrich-Heine-University, Düsseldorf, Germany. Al is the head of the Institute for Health Services Research and Health Economics at the German Diabetes Centre in Düsseldorf and the head of the Institute for Health Services Research and Health Economics at the HeinrichHeine-University, Düsseldorf, Germany.

Ethics approval and consent to participate Not applicable.

Consent for publication

Not applicable.

\section{Competing interests}

Next to his academic affiliation, CMD is employed by Bayer Vital GmbH, Leverkusen, Germany. All other authors declare that they have no competing interests. 


\section{Publisher's Note}

Springer Nature remains neutral with regard to jurisdictional claims in published maps and institutional affiliations.

\section{Author details}

Institute for Health Services Research and Health Economics, Centre for Health and Society, Faculty of Medicine, Heinrich-Heine University Düsseldorf, Moorenstr. 5, 40255 Düsseldorf, Germany. ${ }^{2}$ Institute for Health Services Research and Health Economics, German Diabetes Center (DDZ), Leibniz Center for Diabetes Research at Heinrich Heine University Düsseldorf, Düsseldorf, Germany. ${ }^{3}$ German Center for Diabetes Research (DZD), Neuherberg, Germany.

Received: 9 February 2018 Accepted: 14 November 2018 Published online: 29 November 2018

\section{References}

1. Danaei G, Finucane MM, Lu Y, Singh GM, Cowan MJ, Paciorek CJ, Lin JK Farzadfar F, Khang YH, Stevens GA, et al. National, regional, and global trends in fasting plasma glucose and diabetes prevalence since 1980: systematic analysis of health examination surveys and epidemiological studies with 370 country-years and 2.7 million participants. Lancet (London, England). 2011;378(9785):31-40.

2. International Diabetes Federation. IDF diabetes atlas. 8th ed. Brussels: International Diabetes Federation; 2017.

3. Icks A, Haastert B, Gandjour A, Chernyak N, Rathmann W, Giani G, Rump LC, Trapp R, Koch M. Costs of dialysis--a regional population-based analysis. Nephrol Dial Transplant. 2010;25(5):1647-52.

4. Icks A, Scheer M, Morbach S, Genz J, Haastert B, Giani G, Glaeske G, Hoffmann F. Time-dependent impact of diabetes on mortality in patients after major lower extremity amputation: survival in a population-based 5year cohort in Germany. Diabetes Care. 2011;34(6):1350-4.

5. Icks A, Claessen H, Morbach S, Glaeske G, Hoffmann F. Time-dependent impact of diabetes on mortality in patients with stroke: survival up to 5 years in a health insurance population cohort in Germany. Diabetes Care. 2012;35(9):1868-75.

6. Javaher $\mathrm{P}$, Seidel G, Dierks ML. Participation in disease management of a health insurance company: characteristics and assessment of the process and outcome parameters in the programme. J Public Health. 2006;14(1):37-42.

7. Alberti KG, Zimmet $P$, Shaw J. International Diabetes Federation: a consensus on Type 2 diabetes prevention. Diabet med. 2007:24(5):451-63.

8. Standards of medical care in diabetes--2010. Diabetes care 2010, 33 Suppl 1: S11-S61.

9. Paulweber B, Valensi P, Lindstrom J, Lalic NM, Greaves CJ, McKee M, Kissimova-Skarbek K, Liatis S, Cosson E, Szendroedi J, et al. A European evidence-based guideline for the prevention of type 2 diabetes. Horm metab res. 2010;42(Suppl 1):S3-36.

10. Huang XL, Pan JH, Chen D, Chen J, Chen F, Hu TT. Efficacy of lifestyle interventions in patients with type 2 diabetes: a systematic review and meta-analysis. Eur J intern med. 2016:27:37-47.

11. Lindstrom J, Louheranta A, Mannelin M, Rastas M, Salminen V, Eriksson J, Uusitupa M, Tuomilehto J. The Finnish Diabetes Prevention Study (DPS): lifestyle intervention and 3-year results on diet and physical activity. Diabetes Care. 2003;26(12):3230-6.

12. Knowler WC, Fowler SE, Hamman RF, Christophi CA, Hoffman HJ, Brenneman AT, Brown-Friday JO, Goldberg R, Venditti E, Nathan DM. 10year follow-up of diabetes incidence and weight loss in the Diabetes Prevention Program Outcomes Study. Lancet (London, England). 2009; 374(9702):1677-86

13. Aucott L, Gray D, Rothnie H, Thapa M, Waweru C. Effects of lifestyle interventions and long-term weight loss on lipid outcomes - a systematic review. Obes rev. 2011;12(5):e412-25.

14. Tuomilehto J, Schwarz P, Lindstrom J. Long-term benefits from lifestyle interventions for type 2 diabetes prevention: time to expand the efforts. Diabetes Care. 2011;34(Suppl 2):S210-4

15. Dutton GR, Lewis CE. The Look AHEAD Trial: implications for lifestyle intervention in type 2 diabetes mellitus. Prog Cardiovasc Dis. 2015;58(1):69-75.

16. Jadhav RA, Hazari A, Monterio A, Kumar S, Maiya AG. Effect of physical activity intervention in prediabetes: a systematic review with meta-analysis. J Phys Act Health. 2017;14(9):745-55.
17. Wolf AM, Siadaty M, Yaeger B, Conaway MR, Crowther JQ, Nadler JL, Bovbjerg VE. Effects of lifestyle intervention on health care costs: Improving Control with Activity and Nutrition (ICAN). J Am Diet Assoc. 2007;107(8):1365-73.

18. Aziz Z, Absetz P, Oldroyd J, Pronk NP, Oldenburg B. A systematic review of real-world diabetes prevention programs: learnings from the last 15 years. Implementation science. 2015;10:172

19. Ma J, Yank V, Xiao L, Lavori PW, Wilson SR, Rosas LG, Stafford RS. Translating the Diabetes Prevention Program lifestyle intervention for weight loss into primary care: a randomized trial. JAMA Intern Med. 2013;173(2):113-21.

20. Bouchard DR, Langlois MF, Domingue ME, Brown C, LeBrun V, Baillargeon $J P$. Age differences in expectations and readiness regarding lifestyle modifications in individuals at high risk of diabetes. Arch Phys Med Rehabil. 2012;93(6):1059-64.

21. Horodyska K, Luszczynska A, van den Berg M, Hendriksen M, Roos G, De Bourdeaudhuij I, Brug J. Good practice characteristics of diet and physical activity interventions and policies: an umbrella review. BMC Public Health. 2015;15:19.

22. Knowler WC, Barrett-Connor E, Fowler SE, Hamman RF, Lachin JM, Walker EA, Nathan DM. Reduction in the incidence of type 2 diabetes with lifestyle intervention or metformin. N Engl J Med. 2002;346(6):393-403.

23. James DV, Johnston LH, Crone D, Sidford AH, Gidlow C, Morris C, Foster C. Factors associated with physical activity referral uptake and participation. J Sports Sci. 2008;26(2):217-24

24. Wing RR. Long-term effects of a lifestyle intervention on weight and cardiovascular risk factors in individuals with type 2 diabetes mellitus: four-year results of the Look AHEAD trial. Arch Intern Med. 2010; 170(17):1566-75.

25. CADTH Rapid Response Reports. In: Lifestyle prescriptions: a review of the clinical evidence. edn. Ottawa (ON): Canadian Agency for Drugs and Technologies in Health Copyright (c) 2014 Canadian Agency for Drugs and Technologies in Health.; 2014.

26. Desai JR, Sperl-Hillen JM, O'Connor PJ. Patient preferences in diabetes care: overcoming barriers using new strategies. Journal of comparative effectiveness research. 2013;2(4):351-4.

27. Thomas N, Alder E, Leese GP. Barriers to physical activity in patients with diabetes. Postgrad Med J. 2004;80(943):287-91.

28. Vijan S, Stuart NS, Fitzgerald JT, Ronis DL, Hayward RA, Slater S, Hofer TP. Barriers to following dietary recommendations in Type 2 diabetes. Diabetic medicine. 2005:22(1):32-8

29. Dube MC, Valois P, Prud'homme D, Weisnagel SJ, Lavoie C. Physical activity barriers in diabetes: development and validation of a new scale. Diabetes Res Clin Pract. 2006;72(1):20-7.

30. Lakerveld J, lizelenberg W, van Tulder MW, Hellemans IM, Rauwerda JA, van Rossum AC, Seidell JC. Motives for (not) participating in a lifestyle intervention trial. BMC Med Res Methodol. 2008:8:17.

31. Forbes CC, Plotnikoff RC, Courneya KS, Boule NG. Physical activity preferences and type 2 diabetes: exploring demographic, cognitive, and behavioral differences. The Diabetes educ. 2010;36(5):801-15.

32. Roux L, Ubach C, Donaldson C, Ryan M. Valuing the benefits of weight loss programs: an application of the discrete choice experiment. Obes Res. 2004 12(8):1342-51

33. Owen K, Pettman T, Haas M, Viney R, Misan G. Individual preferences for diet and exercise programmes: changes over a lifestyle intervention and their link with outcomes. Public Health Nutr. 2010;13(2):245-52.

34. McFadden D. Rationality for economists? J Risk Uncertain. 1999;19(1):73-105

35. Alayli-Goebbels AF, Dellaert BG, Knox SA, Ament AJ, Lakerveld J, Bot SD, Nijpels $\mathrm{G}$, Severens JL. Consumer preferences for health and nonhealth outcomes of health promotion: results from a discrete choice experiment. Value in health. 2013;16(1):114-23.

36. Muhlbacher AC, Juhnke C. Patient preferences versus physicians' judgement does it make a difference in healthcare decision making? Appl Health Econ Health Policy. 2013;11(3):163-80.

37. Harrison M, Milbers K, Hudson M, Bansback N. Do patients and health care providers have discordant preferences about which aspects of treatments matter most? Evidence from a systematic review of discrete choice experiments. BMJ Open. 2017;7(5):e014719.

38. Morillas C, Feliciano R, Catalina PF, Ponte C, Botella M, Rodrigues J, Esmatjes E, Lafita J, Lizan L, Llorente I, et al. Patients' and physicians' preferences for type 2 diabetes mellitus treatments in Spain and Portugal: a discrete choice experiment. Patient preference and adherence. 2015;9:1443-58. 
39. Arnolds S, Heckermann S, Koch C, Heissmann N, Sawicki PT: How do patients' preferences compare to the present spectrum of diabetes research? Experimental and clinical endocrinology \& diabetes : official journal, German Society of Endocrinology [and] German Diabetes Association 2013, 121(1):60-63.

40. Arnolds S, Heckermann S, Heise T, Sawicki PT: Spectrum of diabetes research does not reflect patients' scientific preferences: a longitudinal evaluation of diabetes research areas 2010-2013 vs. a cross-sectional survey in patients with diabetes. Exp Clin Endocrinol Diabetes, 123(5):299-302.

41. Ostermann J, Brown DS, de Bekker-Grob EW, Muhlbacher AC, Reed SD. Preferences for health interventions: improving uptake, adherence, and efficiency. The patient. 2017;10(4):511-4.

42. Muhlbacher AC, Johnson FR. Giving patients a meaningful voice in European health technology assessments: the role of health preference research. The patient. 2017;10(4):527-30

43. de Bekker-Grob EW, Berlin C, Levitan B, Raza K, Christoforidi K, Cleemput I, Pelouchova J, Enzmann H, Cook N, Hansson MG. Giving patients' preferences a voice in medical treatment life cycle: the PREFER publicprivate project. The patient. 2017;10(3):263-6.

44. Delamater AM. Improving patient adherence. Clinical Diabetes. 2006;24(2):71-7.

45. Joosten EA, DeFuentes-Merillas $L$, de Weert GH, Sensky T, van der Staak CP, de Jong CA. Systematic review of the effects of shared decision-making on patient satisfaction, treatment adherence and health status. Psychother Psychosom. 2008;77(4):219-26.

46. Mulley AG, Trimble C, Elwyn G. Stop the silent misdiagnosis: patients' preferences matter. BMJ (Clinical research ed). 2012;345:e6572.

47. Weernink MGM, Janus SIM, van Til JA, Raisch DW, van Manen JG, IJzerman MJ. A systematic review to identify the use of preference elicitation methods in healthcare decision making. Pharmaceutical Medicine. 2014;28(4):175-85.

48. Marsh K, IJzerman M, Thokala P, Baltussen R, Boysen M, Kalo Z, Lonngren T, Mussen F, Peacock S, Watkins J, et al. Multiple criteria decision analysis for health care decision making--emerging good practices: report 2 of the ISPOR MCDA emerging good practices task force. Value health. 2016;19(2):125-37.

49. Muhlbacher AC, Kaczynski A. Making good decisions in healthcare with multi-criteria decision analysis: the use, current research and future development of MCDA. Appl Health Econ Health Policy. 2016;14(1):29-40.

50. Marsh K, Lanitis T, Neasham D, Orfanos P, Caro J. Assessing the value of healthcare interventions using multi-criteria decision analysis: a review of the literature. PharmacoEconomics. 2014;32(4):345-65.

51. Adunlin $\mathrm{G}$, Diaby $\mathrm{V}$, Xiao $\mathrm{H}$. Application of multicriteria decision analysis in health care: a systematic review and bibliometric analysis. Health Expect. 2015;18(6):1894-905.

52. Muhlbacher A, Johnson FR. Choice experiments to quantify preferences for health and healthcare: state of the practice. Appl Health Econ Health Policy. 2016;14(3):253-66.

53. Clark MD, Determann D, Petrou S, Moro D, de Bekker-Grob EW. Discrete choice experiments in health economics: a review of the literature. PharmacoEconomics. 2014;32(9):883-902.

54. de Bekker-Grob EW, Ryan M, Gerard K. Discrete choice experiments in health economics: a review of the literature. Health Econ. 2012;21(2):145-72.

55. Quaife M, Terris-Prestholt F, Di Tanna GL, Vickerman P. How well do discrete choice experiments predict health choices? A systematic review and metaanalysis of external validity. Eur J Health. 2018.

56. Salampessy BH, Veldwijk J, Jantine Schuit A, van den Brekel-Dijkstra K, Neslo RE, de Wit Ardine G, Lambooij MS. The predictive value of discrete choice experiments in public health: an exploratory application. The patient. 2015; 8(6):521-9.

57. Liberatore MJ, Nydick RL. The analytic hierarchy process in medical and health care decision making: a literature review. Eur J Oper Res. 2008;189(1):194-207.

58. Danner M, Hummel JM, Volz F, van Manen JG, Wiegard B, Dintsios CM, Bastian $H$, Gerber A, ljzerman MJ. Integrating patients' views into health technology assessment: analytic hierarchy process (AHP) as a method to elicit patient preferences. Int J Technol Assess Health Care. 2011; 27(4):369-75.

59. Hummel MJ, Volz F, van Manen JG, Danner M, Dintsios CM, ljzerman MJ, Gerber A. Using the analytic hierarchy process to elicit patient preferences: prioritizing multiple outcome measures of antidepressant drug treatment. The patient. 2012;5(4):225-37.

60. Maruthur NM, Joy SM, Dolan JG, Shihab HM, Singh S. Use of the analytic hierarchy process for medication decision-making in type 2 diabetes. PLoS One. 2015;10(5):e0126625.
61. Joy SM, Little E, Maruthur NM, Purnell TS, Bridges JF. Patient preferences for the treatment of type 2 diabetes: a scoping review. PharmacoEconomics. 2013;31(10):877-92.

62. Gelhorn HL, Stringer SM, Brooks A, Thompson C, Monz BU, Boye KS, Hach T, Lund SS, Palencia R. Preferences for medication attributes among patients with type 2 diabetes mellitus in the UK. Diabetes Obes Metab. 2013;15(9): 802-9.

63. Purnell TS, Joy S, Little E, Bridges JF, Maruthur N. Patient preferences for noninsulin diabetes medications: a systematic review. Diabetes Care. 2014 37(7):2055-62.

64. von Arx LB, Kjeer T. The patient perspective of diabetes care: a systematic review of stated preference research. The patient. 2014;7(3):283-300.

65. Muhlbacher AC, Kaczynski A. Patients' preferences in the medicamentous treatment of diabetes mellitus type 2: a systematic classification and metacomparison of patient preference studies. Bundesgesundheitsblatt, Gesundheitsforschung, Gesundheitsschutz. 2015;58(4-5):452-66.

66. Muhlbacher A, Bethge S. What matters in type 2 diabetes mellitus oral treatment? A discrete choice experiment to evaluate patient preferences. Eur J Health Econ. 2016;17(9):1125-40.

67. Flood EM, Bell KF, de la Cruz MC, Ginchereau-Sowell FM. Patient preferences for diabetes treatment attributes and drug classes. Curr Med Res Opin. 2017 33(2):261-8

68. Thrasher J. Pharmacologic management of type 2 diabetes mellitus: available therapies. Am J Med. 2017;130(6s):S4-s17.

69. Johnson FR, Manjunath R, Mansfield CA, Clayton LJ, Hoerger TJ, Zhang P. High-risk individuals' willingness to pay for diabetes risk-reduction programs. Diabetes Care. 2006;29(6):1351-6.

70. Veldwijk J, Lambooij MS, van Gils PF, Struijs JN, Smit HA, de Wit GA. Type 2 diabetes patients' preferences and willingness to pay for lifestyle programs: a discrete choice experiment. BMC Public Health. 2013;13:1099.

71. Liberati A, Altman DG, Tetzlaff J, Mulrow C, Gotzsche PC, loannidis JP, Clarke M, Devereaux PJ, Kleijnen J, Moher D. The PRISMA statement for reporting systematic reviews and meta-analyses of studies that evaluate health care interventions: explanation and elaboration. Ann Intern Med. 2009;151(4): W65-94.

72. Moher D, Shamseer L, Clarke M, Ghersi D, Liberati A, Petticrew M, Shekelle P, Stewart LA. Preferred reporting items for systematic review and metaanalysis protocols (PRISMA-P) 2015 statement. Systematic reviews. 2015;4:1.

73. van Hoorn R, Kievit W, Booth A, Mozygemba K, Lysdahl KB, Refolo P, Sacchini D, Gerhardus A, van der Wilt GJ, Tummers M. The development of PubMed search strategies for patient preferences for treatment outcomes. BMC Med Res Methodol. 2016;16:88.

74. Thokala P, Devlin N, Marsh K, Baltussen R, Boysen M, Kalo Z, Longrenn T, Mussen F, Peacock S, Watkins J, et al. Multiple criteria decision analysis for health care decision making--an introduction: report 1 of the ISPOR MCDA emerging good practices task force. Value health. 2016;19(1):1-13.

75. Hauber AB, Gonzalez JM, Groothuis-Oudshoorn CG, Prior T, Marshall DA, Cunningham C, IJzerman MJ, Bridges JF. Statistical methods for the analysis of discrete choice experiments: a report of the ISPOR conjoint analysis good research practices task force. Value health. 2016;19(4):300-15.

76. Bridges JF, Hauber AB, Marshall D, Lloyd A, Prosser LA, Regier DA, Johnson FR, Mauskopf J. Conjoint analysis applications in health--a checklist: a report of the ISPOR good research practices for conjoint analysis task force. Value Health. 2011;14(4):403-13.

77. Reed Johnson F, Lancsar E, Marshall D, Kilambi V, Muhlbacher A, Regier DA, Bresnahan BW, Kanninen B, Bridges JF. Constructing experimental designs for discrete-choice experiments: report of the ISPOR conjoint analysis experimental design good research practices task force. Value health. 2013; 16(1):3-13.

78. Lancsar E, Louviere J. Conducting discrete choice experiments to inform healthcare decision making: a user's guide. PharmacoEconomics. 2008; 26(8):661-77

79. Schmidt K, Aumann I, Hollander I, Damm K, von der Schulenburg JM. Applying the analytic hierarchy process in healthcare research: a systematic literature review and evaluation of reporting. BMC Med Inform Decis Mak. 2015:15:112. 